\title{
PARP Inhibitors in Reproductive System Cancers: Current Use and Developments
}

\author{
Geraldine O’Sullivan Coyne ${ }^{1} \cdot$ Alice P. Chen ${ }^{1} \cdot$ Robert Meehan $^{1} \cdot$ James H. Doroshow $^{1,2}$
}

Published online: 11 January 2017

(c) The Author(s) 2017. This article is published with open access at Springerlink.com

\begin{abstract}
The repair of DNA damage is a critical cellular process governed by multiple biochemical pathways that are often found to be defective in cancer cells. The poly(ADP-ribose) polymerase (PARP) family of proteins controls response to single-strand DNA breaks by detecting these damaged sites and recruiting the proper factors for repair. Blocking this pathway forces cells to utilize complementary mechanisms to repair DNA damage. While PARP inhibition may not, in itself, be sufficient to cause tumor cell death, inhibition of DNA repair with PARP inhibitors is an effective cytotoxic strategy when it is used in patients who carry other defective DNA-repair mechanisms, such as mutations in the genes BRCA 1 and 2. This discovery has supported the development of PARP inhibitors (PARPi), agents that have proven effective against various types of tumors that carry BRCA mutations. With the application of next-generation sequencing of tumors, there is increased interest in looking beyond $B R C A$ mutations to identify genetic and epigenetic aberrations that might lead to similar defects in DNA repair, conferring susceptibility to PARP inhibition. Identification of these genetic lesions and the development of screening assays for their detection may allow for the selection of patients most likely to respond to this class of anticancer agents. This article provides an overview of clinical trial results
\end{abstract}

James H. Doroshow

doroshoj@mail.nih.gov

1 Early Clinical Trials Development Program, Division of Cancer Treatment and Diagnosis, National Cancer Institute, National Institutes of Health, 31 Center Drive, Room 3A44, Bethesda, MD 20892, USA

2 Developmental Therapeutics Branch, Center for Cancer Research, National Cancer Institute, National Institutes of Health, Bethesda, MD 20892, USA obtained with PARPi and describes the companion diagnostic assays being established for patient selection. In addition, we review known mechanisms for resistance to PARPi and potential strategies for combining these agents with other types of therapy.

\section{Key Points}

PARP inhibition is a highly effective approach to the treatment of ovarian cancers caused by specific aberrations in DNA repair genes; this approach has led to the successful regulatory approval of olaparib, rucaparib, and niraparib for patients with advanced ovarian cancer.

The continuing development of effective companion diagnostic testing to identify patients most likely to respond to PARP inhibition will improve the therapeutic index of this drug class in the future.

\section{Introduction}

The human DNA damage-response (DDR) system encompasses a network of cellular proteins designed to detect and repair DNA breaks with the intent of maintaining genomic integrity [1]. Unrepaired DNA damage can lead to genetic mutations, resulting in malignant transformation. Our growing understanding of the DDR process and the mechanisms that govern DNA repair has provided novel targets for anticancer therapies.

It has been more than half a century since the discovery of the PARP [poly(ADP-ribose) polymerase]-1 enzyme 
and 30 years since the development of a prototype PARP inhibitor (PARPi) 3-aminobenzamide (3AB) [2]. PARP-1, which remains the best described of the super family of PARP proteins, controls the repair of single-strand breaks (SSBs) in DNA through the base excision repair pathway (BER). PARPi effectively eliminate a cell's capacity to repair SSBs through the BER, forcing the cell to instead rely upon other DNA-repair mechanisms, specifically homologous recombination (HR) and the non-homologous end joining (NHEJ) pathways [3, 4]. However, cells deficient in BRCA 1 and 2, two of the most studied genes involved in HR, are unable to fully utilize the HR pathway and die from accumulated unrepaired DNA breaks when exposed to PARPi, exemplifying the concept of cancerspecific synthetic lethality (see Polyak and Garber [5] and Kaelin [6] for reviews). Mutations in BRCA, which can be inherited through the germline or can occur de novo in somatic cells, contribute to genetic instability and are most commonly associated with breast and ovarian cancers [7].

From preclinical research — and most recently, from clinical proof-of-concept studies-we now know that inability to undergo HR, or HR deficiency (HRD), is not solely defined by deleterious BRCA 1 and 2 mutations but also by genomic alterations and/or epigenetic silencing of other pathway genes, including ATR, ATM, RAD51/54, CHK1/2, NBS1, PTEN, and $P A L B 2$. These genetic and epigenetic aberrations confer socalled 'BRCAness,' or susceptibility to DNA-damaging treatments similar to that seen with BRCA deficiency, to affected cells and render them sensitive to PARPi. The association of the BRCAness phenotype with a wider range of genetic mutations may expand the utility of PARPi beyond reproductive malignancies, the tumor types for which these agents were originally intended $[8,9]$.

This encouraging but complex area of study has fortunately overcome initial disappointment caused by the failure of the reportedly first-in-class PARPi, iniparib (BSI201; Sanofi-Aventis, Paris, France). Development of iniparib was halted at an advanced stage following an interim negative efficacy analysis of a pivotal combination phase III trial in advanced triple negative breast cancer (TNBC) in 2011 [10, 11]. Many reasons have been postulated for the discrepancy between this trial and a phase II trial of the same combination; however, the small size of the phase II trial and the definitive demonstration that iniparib does not in fact inhibit PARP are the most likely explanations for this apparent incongruity $[7,9]$.

With the advent of targeted anticancer therapy, next-generation molecular sequencing, and genetic profiling, as well as the recent finding that HRD is related to more than alterations in the function of $B R C A$ genes, there is now an increased focus on determining which genomic markers can clinically define the patient populations most likely to benefit from treatment with PARPi. Currently, five PARPi are actively progressing through clinical development: olaparib (AZD2281, Ku0059436, Lymparza ${ }^{\circledR}$; AstraZeneca, Rockville, MD, USA), veliparib (ABT 888; AbbVie, North Chicago, IL, USA), niraparib (MK-4827; Tesaro, Waltham, MA, USA), rucaparib (PF-01367338, AG01469, CO-338, Rubraca ; Clovis Oncology, Boulder, CO, USA), and talazoparib (BMN 673; Medivation, San Francisco, CA, USA) (Table 1). Sequencing-based companion diagnostic (CDx) testing for PARPi is being developed in parallel, reflecting the increased focus on determining clinically meaningful and predictive genomic markers that can define the patient populations most likely to respond to these agents. This review focuses on clinical results of PARPi in reproductive cancers and selected data from nonreproductive tumor types as well as on strategies for patient selection and combination treatment.

\section{Mechanisms of Action}

The PARP family of proteins includes at least 17 enzymes, with PARP-1 described as the most abundant in normal tissues. Only two members of this enzyme family, PARP-1 and PARP-2, are known to be involved in DNA damage repair, and PARP-1 accounts for at least $85 \%$ of cellular PARP activity [12]. It has been suggested that PARP-3 plays a role in the DDR as well, in part by controlling PARP-1 activity through heterodimerization [13, 14]. Under normal circumstances, the main function of PARP is to sense SSBs and recruit SSB repair proteins to the damaged chromatin site through enzymatic activity [15-17]. Following binding of these proteins, the PARP enzyme undergoes ADP-ribosylation and, partnering with H1/H2B histones, permits uncoiling of the chromatin for repair [8]. This process typically requires the consumption of $\mathrm{NAD}^{+}$and the release of nicotinamide, the process that is the primary target for PARP inhibition; as a result, PARPi are defined as b-nicotinamide adenine dinucleotide $\left(\mathrm{NAD}^{+}\right)$-competitive inhibitors $[8,18]$.

However, a secondary effect of these agents is PARP 'trapping,' a phenomenon in which, as a consequence of enzyme inhibition by the PARPi, PARP itself does not disengage from the damaged chromatin site. The trapping of PARP at damaged DNA sites leads not only to unrepaired SSBs but also to the generation and persistence of secondary double-strand breaks (DSBs) during S-phase, which require HR for repair. The trapped PARP makes chromatin inaccessible to other repair proteins, further promoting sustained DNA damage [4, 19, 20]. PARP trapping represents an additional mechanism of action for PARPi, and there are preclinical data to suggest that the consequences of trapped PARP-DNA complexes are actually more cytotoxic than the unrepaired SSBs that initially result from the loss of PARP activity [20]. 
Table 1 Summary table of PARP inhibitors approved or in clinical development for the treatment of reproductive system cancers

\begin{tabular}{|c|c|c|c|c|c|c|}
\hline $\begin{array}{l}\text { Drug } \\
\text { (company) }\end{array}$ & $\begin{array}{l}\text { Molecular } \\
\text { targets }\end{array}$ & Dose & Common AEs & $\begin{array}{l}\text { Potential drug } \\
\text { interactions }^{\mathrm{a}}\end{array}$ & Regulatory approval & Indication \\
\hline \multirow[t]{2}{*}{$\begin{array}{l}\text { Olaparib; } \\
\text { Lynparza }^{\mathrm{TM}} \\
\text { (AstraZeneca) }\end{array}$} & \multirow[t]{2}{*}{ PARP-1, -2 } & \multirow[t]{2}{*}{$\begin{array}{l}400 \mathrm{mg} \\
\text { bid } \\
\text { (oral) }\end{array}$} & \multirow[t]{2}{*}{$\begin{array}{l}\text { Nausea }(59-78 \%), \\
\text { fatigue }(41-65 \%) \text {, } \\
\text { vomiting } \\
(34-50 \%), \text { anemia } \\
(12-32 \%)\end{array}$} & \multirow[t]{2}{*}{$\begin{array}{l}\text { Strong and } \\
\text { moderate } \\
\text { CYP3A } \\
\text { inhibitors and } \\
\text { inducers, myelo- } \\
\text { suppressive } \\
\text { agents }\end{array}$} & $\begin{array}{l}\text { FDA approved } \\
\text { (accelerated } \\
\text { approval) and } \\
\text { EMA approved, } \\
\text { 12/2014 }\end{array}$ & $\begin{array}{l}\text { FDA: treatment of germline } \\
B R C A \text {-mutated ovarian } \\
\text { cancer after } \geq 3 \text { prior lines } \\
\text { of therapy } \\
\text { EMA: maintenance } \\
\text { treatment of platinum- } \\
\text { sensitive relapsed } B R C A- \\
\text { mutated (germline and/or } \\
\text { somatic) high-grade } \\
\text { serous epithelial ovarian, } \\
\text { fallopian tube, or primary } \\
\text { peritoneal cancer in } \\
\text { complete or partial } \\
\text { response to platinum- } \\
\text { based chemotherapy }\end{array}$ \\
\hline & & & & & $\begin{array}{l}\text { FDA breakthrough } \\
\text { designation, } \\
01 / 2016\end{array}$ & $\begin{array}{l}\text { BRCA- or ATM-mutated } \\
\text { metastatic, castration- } \\
\text { resistant prostate cancer in } \\
\text { patients who have } \\
\text { progressed on prior } \\
\text { taxane-based } \\
\text { chemotherapy and at least } \\
\text { one newer hormonal agent } \\
\text { (abiraterone or } \\
\text { enzalutamide) }\end{array}$ \\
\hline $\begin{array}{l}\text { Veliparib } \\
\quad \text { (Abbvie) }\end{array}$ & PARP-1, -2 & $\begin{array}{l}400 \mathrm{mg} \\
\text { bid } \\
\text { (oral) }\end{array}$ & $\begin{array}{c}\text { Fatigue }(6 \%) \text {, nausea } \\
(4 \%), \text { leukopenia } \\
(2 \%) \text { [phase II data] }\end{array}$ & - & - & - \\
\hline $\begin{array}{l}\text { Niraparib } \\
\text { (Tesaro) }\end{array}$ & PARP-1, -2 & $\begin{array}{l}300 \mathrm{mg} \\
\text { od } \\
(\text { oral })\end{array}$ & $\begin{array}{l}\text { Thrombocytopenia } \\
(33.8 \%), \text { anemia } \\
(25.3 \%), \\
\text { neutropenia } \\
(19.6 \%) \text { [phase III } \\
\text { data] }\end{array}$ & $\begin{array}{l}\text { Drugs } \\
\text { metabolized by } \\
\text { CYP1A2, P-gp } \\
\text { inhibitors or } \\
\text { substrates, } \\
\text { anticoagulants } \\
\text { and antiplatelets }\end{array}$ & $\begin{array}{l}\text { FDA fast-track } \\
\text { designation, } \\
\text { 09/2016; NDA } \\
\text { submission to } \\
\text { FDA and MAA } \\
\text { submission to } \\
\text { EMA, 10/2016 }\end{array}$ & $\begin{array}{l}\text { Maintenance treatment for } \\
\text { platinum-sensitive, } \\
\text { recurrent epithelial } \\
\text { ovarian, fallopian tube, or } \\
\text { primary peritoneal cancer } \\
\text { in response to platinum- } \\
\text { based chemotherapy; } \\
\text { regardless of } B R C A \text { and } \\
\text { HRD status }\end{array}$ \\
\hline $\begin{array}{l}\text { Rucaparib; } \\
\text { Rubraca } \\
\text { (Clovis } \\
\text { oncology) }\end{array}$ & $\begin{array}{l}\text { PARP-1, -2, - } \\
\text { 3; also } \\
\text { inhibits } \\
\text { PARP-4, - } \\
\text { 12, -15, -16, } \\
\text { and } \\
\text { tankyrase } 1 \\
\text { and } 2\end{array}$ & $\begin{array}{l}600 \mathrm{mg} \\
\text { bid } \\
\text { (oral) }\end{array}$ & $\begin{array}{l}\text { Occuring } \geq 15 \% \text { : } \\
\text { nausea, } \\
\text { asthenia/fatigue, } \\
\text { anemia, transient } \\
\text { ALT/AST } \\
\text { elevations (grade } \\
\text { 1/2) [phase II data] }\end{array}$ & - & $\begin{array}{l}\text { FDA-approved } \\
\quad \text { (accelerated } \\
\text { approval), 12/2016 }\end{array}$ & $\begin{array}{l}\text { Treatment of advanced } \\
B R C A \text {-mutated (germline } \\
\text { and/or somatic) ovarian } \\
\text { cancer after two or more } \\
\text { lines of prior } \\
\text { chemotherapy }\end{array}$ \\
\hline $\begin{array}{l}\text { Talazoparib } \\
\text { (Medivation) }\end{array}$ & PARP-1, -2 & $\begin{array}{l}1 \mathrm{mg} \text { od } \\
\text { (oral) }\end{array}$ & $\begin{array}{l}\text { Fatigue, nausea, } \\
\text { alopecia, } \\
\text { thrombocytopenia } \\
\text { [phase I data] }\end{array}$ & $\begin{array}{l}\text { Anticoagulants } \\
\text { and antiplatelets }\end{array}$ & - & - \\
\hline
\end{tabular}

$A E$ adverse event, $A L T$ alanine transaminase ratio, $A S T$ aspartate transaminase, bid twice daily, $C Y P$ cytochrome P450, EMA European Medicines Agency, $H R D$ homologous recombination deficiency, MAA marketing authorization application, NDA new drug application, od once daily, $P$-gp P-glycoprotein

${ }^{a}$ Not an exhaustive list; more studies are needed regarding PARPi drug-drug interactions

Though preclinical data would suggest that the more effective PARPi are those that most strongly trap PARPDNA complexes [21, 22], clinical efficacy has yet to correlate with preclinical potency in the clinical trials of these agents. Moreover, it is unclear whether PARP trapping is actually necessary for clinical efficacy. 
Pharmacodynamic markers of PARP inhibition have been helpful in the initial development of PARPi, providing clinical evidence of mechanism of action. However, correlations between the extent of PARP inhibition and clinical activity have yet to be demonstrated. There is no clinical assay at present for evaluation of PARP trapping.

Extensive preclinical investigative studies have attempted to elucidate the genomic and cellular effects of PARP enzymatic inhibition in tumor cells. These studies initially demonstrated the synthetic lethal interaction between PARP and BRCA. Synthetic lethality is defined as the phenomenon in which the combination of two nonlethal defects leads to cell death [5, 23, 24]. Specifically, $B R C A$-mutated tumor cells, which are unable to undergo $\mathrm{HR}$, are effectively killed by the unrepaired DNA damage induced by the loss of PARP activity [19, 25]. Ongoing studies of the range of novel synthetic lethal interactions that can be produced by combinations of PARPi and drugs that damage DNA by interfering with other DNA-repair pathways are likely to prompt a steady flow of new DDRfocused clinical trials based on synthetic lethal combinations.

\section{Clinical Development of PARP Inhibitors (PARPi)}

\subsection{Clinical Studies of PARPi}

\subsubsection{Olaparib}

Olaparib has undergone extensive preclinical and clinical testing and was the first PARPi to receive accelerated approval by the US FDA for treatment of germline BRCA 1 and 2-mutated $(\mathrm{g} B R C A 1 / 2 \mathrm{~m})$ advanced ovarian cancer. FDA approval coincided with a first approval for an in vitro CDx test for this drug class, BRACAnalysis CDx ${ }^{\circledR}$ (Myriad Genetics, Inc., Salt Lake City, UT, USA), to detect the presence of $g B R C A \mathrm{~m}$, identified by polymerase chain reaction (PCR)/Sanger sequencing of whole blood specimens. Olaparib is also the first PARPi to be approved by the European Medicines Agency (EMA), though with a different indication (maintenance therapy for patients with $\mathrm{g} B R C A \mathrm{~m}$-associated ovarian cancer following platinumbased chemotherapy).

A proof-of-concept phase I trial evaluating olaparib (PARP1/2 drug concentration producing 50\% inhibition $\left.\left[\mathrm{IC}_{50}\right] 5 \mathrm{nM} / 1 \mathrm{nM}\right)$ demonstrated antitumor activity and defined the maximum tolerated dose (MTD) as $400 \mathrm{mg}$ twice daily (bid) $[22,26]$. A subsequent 50-patient expansion cohort of individuals with $\mathrm{g} B R C A \mathrm{~m}$-associated ovarian tumors confirmed that olaparib provides clinical benefit for this target population. There also was an association between platinum sensitivity and PARPi response (response rate $[R R] 46,34$, and $0 \%$ for the platinum-sensitive, -resistant, and -refractory groups, respectively) that suggested a possible crossover mechanism of resistance between platinum and olaparib [27]. Two separate phase II trials then provided further support for the efficacy of olaparib in patients with $\mathrm{g} B R C A \mathrm{~m}$-associated advanced breast and ovarian cancers [28, 29]. A follow-up phase II trial that evaluated olaparib in high-grade serous ovarian cancer (HGSOC) and TNBC, in which activity was assessed for tumors with 'BRCAness' regardless of true $B R C A$ status, reported an RR of $23 \%$ in non-gBRCAm. This suggests that, for patients with HGSOC, a percentage of tumors express a phenotype consistent with 'BRCAness'. Though no responses were observed in patients with breast cancer, those with breast cancer carrying $B R C A$ mutations had more unconfirmed partial responses (PRs) [30].

Olaparib was also compared with pegylated doxorubicin (PLD), an agent commonly used in the treatment of platinum-resistant ovarian cancer. In patients with recurrent $\mathrm{g} B R C A \mathrm{~m}$ ovarian cancer, there was no significant difference between median progression-free survival (PFS) with olaparib versus that with PLD. However, it is important to note that the efficacy of PLD in this trial was greater than expected. Furthermore, the fact that this well-tolerated oral PARPi led to a survival rate similar to that seen with intravenous chemotherapy was a promising outcome [31], though the study overall was viewed to have a negative result.

As previously mentioned, olaparib is approved by the EMA as monotherapy for the maintenance treatment of adult patients with platinum-sensitive relapsed $B R C A$-mutated high-grade serous epithelial ovarian, fallopian tube, or primary peritoneal cancer who are in complete response or who experienced PRs with platinum-based chemotherapy. This approval was based, in part, on a placebo-controlled phase II maintenance trial in patients with platinumsensitive recurrent HGSOC (with or without $\mathrm{g} B R C A \mathrm{~m}$ ). Although the modest improvement in PFS (8.4 months with olaparib $400 \mathrm{mg}$ vs. 4.8 with placebo) was at first not expected to translate into a significant overall survival (OS) benefit, a pre-planned retrospective review of the trial revealed that patients carrying $\mathrm{g} B R C A \mathrm{~m}$ showed an improvement in PFS with olaparib treatment [32, 33]. Interestingly, following the presentation of the initial PFS results in 2011 at the American Society of Clinical Oncology (ASCO) annual meeting, AstraZeneca announced that they would not continue development of olaparib for HGSOC because, as mentioned above, the PFS gains were not expected to lead to OS improvement [3]. However, the company reversed this decision after the release of the results of the pre-planned retrospective 
analysis based on gBRCAm status at the 2013 ASCO annual meeting. In this pre-planned retrospective analysis of PFS, of the 131 patients receiving olaparib and the 123 patients receiving placebo, 56 and $50 \%$ of patients had a deleterious (or suspected deleterious) germline mutation in $B R C A$. Germline BRCA mutation carriers had a difference in PFS of 11.2 months compared with 4.3 months in patients receiving olaparib versus placebo (hazard ratio [HR] $0.18 ; 95 \%$ confidence interval [CI] 0.10-0.31; $p=0.0001$ ) [33]. Additionally, based on an unplanned retrospective exploratory subgroup analysis, patients with sporadic ovarian cancer also had an improvement in PFS of 7.4 versus 5.5 months (HR 0.54 ; 95\% CI $0.34-0.85$; $p=0.0075)$. Notably, there was no difference in OS in either subgroup [33].

Despite these results and the approval granted by the EMA, the Oncologic Drugs Advisory Committee (ODAC) voted against FDA approval of olaparib as maintenance therapy in platinum-sensitive relapsed ovarian cancer, citing concerns regarding therapy-associated toxicities [34]. However, the FDA did grant accelerated approval of olaparib as a single agent in patients with $\mathrm{g} B R C A \mathrm{~m} 1 / 2$-associated ovarian cancer after three or more prior lines of therapy, based on encouraging phase II results showing that $34 \%$ of patients had objective responses that lasted an average of 7.9 months. Two AstraZeneca-sponsored randomized phase III trials are now collecting additional data on olaparib monotherapy as a maintenance treatment: SOLO1 (NCT01844986) and SOLO2 (NCT01874353). These trials were initiated in June 2013 in patients with ovarian cancer with a gBRCAm. SOLO1 enrolled patients with newly diagnosed advanced (FIGO [International Federation of Gynecology and Obstetrics] stage III-IV) disease that has responded to first-line platinum therapy. For SOLO2, a similar patient population had to have completed at least two lines of platinum therapy. AstraZeneca has recently announced that SOLO2 has met its primary endpoint, PFS, and reportedly exceeded that observed in the prior phase II maintenance study in patients with platinum-sensitive relapsed ovarian cancer [35]. However, final approval of olaparib is conditional on the results of the SOLO3 phase III trial (olaparib vs. physician treatment of choice), which also uses PFS as the primary outcome.

Of note, olaparib has also received an FDA breakthrough therapy designation for patients with $B R C A$ - or Ataxia-telangiectasia mutated (ATM)-mutated metastatic castration-resistant prostate cancer (mCRPC) who have progressed on prior taxane-based chemotherapy and at least one newer hormonal agent (abiraterone or enzalutamide) [36]. These patients with prostate cancer must also have been treated with either of two hormonal agents: enzalutamide or abiraterone acetate. The designation is based on data from the open-label single-arm phase II TOPARP-A trial (NCT01682772). Olaparib monotherapy had an overall response rate (ORR [complete response + PR]) of $88 \%$ in a biomarker-defined subgroup of patients who had DNA-repair defects (including BRCA2 mutations, $A T M$ mutations, somatic deletions of $B R C A l$ or CHEK2 in FANCA deletions with no germline events). Further results from this study are eagerly awaited.

\subsubsection{Veliparib}

Veliparib, an oral PARP-1 and -2 inhibitor (PARP1/2 inhibition constants $\left[K_{\mathrm{i}}\right] 5.2 / 2.9 \mathrm{nM}$ ), is currently undergoing extensive clinical development, with ongoing and reported trials for a number of combinations and histologies [22]. The first single-agent phase I/II trial in patients with either $\mathrm{g} B R C A \mathrm{~m}$ or $B R C A$-platinum-refractory ovarian cancer and basal-like breast cancer (BRCA-wild type [WT]) reported greater clinical activity in those with $\mathrm{g} B R C A \mathrm{~m}$ disease than in those with WT disease (ORR of $23 \%$ combining all dose levels) and demonstrated a tolerable toxicity profile [37]. The recommended phase II dose (RP2D) was established as oral administration $400 \mathrm{mg}$ bid. At this dose level, 28 patients with $\mathrm{g} B R C A \mathrm{~m}$ disease were evaluable, with an ORR of $40 \%$ and a clinical benefit rate (CBR [complete response $+\mathrm{PR}+$ stable disease]) of $68 \%$. In total, 24 patients with BRCA WT disease (21 breast and 3 ovary) were also evaluable for response and had an ORR of $4 \%$ and a CBR of $38 \%$. A phase II study of single-agent veliparib targeting HGSOC demonstrated the efficacy of veliparib as a monotherapy against platinumsensitive and platinum-resistant $\mathrm{g} B R C A \mathrm{~m}$ ovarian cancer [38].

Veliparib has been considered the PARPi most suitable for combination studies because of its modest hematopoietic toxicity. Combination trials have been reported with cyclophosphamide, temozolomide, carboplatin, and mitomycin C. Most recently, the I-SPY (Investigation of Serial Studies to Predict Your Therapeutic Response through Imaging and Molecular Analysis 2) trial initiative published encouraging results for veliparib in combination with carboplatin in the neoadjuvant setting for patients with early-stage breast cancer [39]. This prospective phase II adaptive design study is screening multiple experimental combinations using a predefined biomarker 'signature' panel (based on expression of the human epidermal growth factor-2 [HER-2], hormone receptors, and a 70-gene assay) that generates a distinct biomarker combination of ten 'signatures'. The primary endpoint of the study is pathological complete response (pCR). Patients were randomly assigned to receive veliparib plus carboplatin $(n=72)$ or control therapy $(n=44)$. At the completion of chemotherapy, the estimated rates of pCR in the 
triple-negative population were $51 \% \quad(95 \%$ Bayesian probability interval $[\mathrm{PI}] 36-66)$ in the veliparib plus carboplatin group versus $26 \%$ (95\% PI 9-43) in the control group. However, this trial was not designed to predict improvement in disease-free or OS with the veliparib and carboplatin combination in phase III neoadjuvant trials but rather to predict a positive result with the use of $\mathrm{pCR}$ rate as an endpoint. Though this surrogate marker has been associated with a decrease in death, it has not always been consistent with an improvement in long-term outcomes $[40,41]$. Also, the trial did not specifically address the benefit of adding veliparib but rather only the combination of veliparib with carboplatin, as no randomized comparison with single-agent carboplatin was included [42]. Veliparib continues to be studied in a variety of ovarian and cervical cancer settings.

\subsubsection{Niraparib}

Niraparib (MK-4827) is another selective PARP-1 and -2 inhibitor (PARP1/2 $K_{\mathrm{i}} 3.2 / 4.0 \mathrm{nM}$ ) [22]. A phase I/Ib doseescalation study in 100 patients with advanced solid tumors established $300 \mathrm{mg} /$ day as the MTD and reported confirmed PRs in 8 of $20(40 \%) \mathrm{g} B R C A \mathrm{~m}$-associated ovarian or primary peritoneal cancers, with response rates of $50 \%$ in platinum-sensitive and $33 \%$ in platinum-resistant disease $[43,44]$. Durable PRs were also observed in sporadic HGSOC (67\% of patients [two of three] with platinumsensitive disease and $16 \%$ of patients [3 of 19] with platinum-resistant disease responded). Other interesting responses from the trial included a single patient with $\mathrm{g} B R C A \mathrm{~m} 2$ non-small-cell lung cancer who had stable disease for 175 days and 9 of 21 patients (43\%) with CRPC who had stable disease for a median duration of 254 days [44].

In September 2016, niraparib received FDA fast-track designation for treatment of patients with recurrent platinum-sensitive ovarian, fallopian tube, or primary peritoneal cancer based on the results of the phase III ENGOT (European Network for Gynecological Oncological Trial)OV16/NOVA trial, which met its primary endpoint [9]. The NOVA trial examined the efficacy and safety of niraparib compared with placebo as maintenance treatment in a broad population of patients with platinum-sensitive recurrent ovarian cancer, with a primary endpoint of PFS. Two separate cohorts ( $\mathrm{g} B R C A \mathrm{~m}$ and non-gBRCAm) were enrolled, with all patients having mutational status determined using centralized BRACAnalysis testing. Patients were randomly assigned in a 2:1 ratio to receive niraparib or placebo once daily (od) in 28-day cycles until disease progression. The myChoice HRD test (Myriad Genetics) was performed on archived tumor tissue samples and used to further classify the population of patients in the non- $\mathrm{g} B R C A \mathrm{~m}$ cohort as either HRD positive or negative. The trial enrolled 553 patients, with 203 in the $\mathrm{g} B R C A \mathrm{~m}$ cohort (138 assigned to niraparib and 65 to placebo) and 350 patients in the non-gBRCAm cohort (234 assigned to niraparib and 116 to placebo). Patients in the niraparib group had a significantly longer median duration of PFS than those in the placebo group, irrespective of $\mathrm{g} B R C A \mathrm{~m}$ or HRD status (21.0 vs. 5.5 months in the $\mathrm{g} B R C A \mathrm{~m}$ cohort [HR 0.27 ; $95 \%$ CI $0.17-0.41$ ], 12.9 vs. 3.8 months in the non-gBRCAm HRD-positive cohort [HR $0.38 ; 95 \% \mathrm{CI}$ $0.24-0.59$ ], and 9.3 vs. 3.9 months in the overall non$\mathrm{g} B R C A \mathrm{~m}$ cohort [HR 0.45 ; 95\% CI $0.34-0.61$ ] $; p<0.001$ for all three comparisons) [9]. In an exploratory subgroup analysis of the HRD-negative cohort, niraparib also significantly improved PFS (6.9 vs. 3.8 months; HR 0.58; $95 \%$ CI $0.36-0.92 ; p=0.02$ ). While patients with gBRCA mutations derived the greatest benefit from niraparib treatment, these results demonstrate that efficacy of niraparib is independent of $g B R C A$ mutations and HRD status. Furthermore, the results of the NOVA trial show striking durability of response with niraparib; even in the HRDnegative subgroup, a percentage of patients had sustained long-term benefit and remained free of progression at 18 months. Additional studies will likely aim to elucidate the mechanism driving long-term response to niraparib in non- $g B R C A m$ HRD-negative patients and discover the reason for the lack of benefit in the minority of HRDpositive patients who did not respond.

Further research is also needed to identify ways to predict which patients will respond to niraparib treatment, either in the short or the long term ( $>18$ months). Speculatively, non-HRD factors-such as amplification of CCNE1 (which encodes cyclin E1, a cell cycle regulator)may serve as predictive biomarkers. The cancer genome atlas (TCGA) project reported the mutational spectrum of HGSOC and found this tumor type to be defined by widespread loss of p53 (which plays a role in modulating HR) as well as germline and sporadic BRCA mutations, with approximately $50 \%$ of all tumors having associated defects in the HR pathway [45, 46]. Analysis of HGSOC tumors without HRD showed that around 20\% have amplification of $C C N E 1$, which is associated with aggressive disease and reduced OS. It has been suggested that $C C N E 1$ amplification and BRCAI loss are a synthetic lethal combination, explaining the mutual exclusivity of these genetic alterations [47]. CCNE1 amplification reduces tolerability for BRCA loss and may also play a role in PARPi sensitivity.

Tesaro submitted a rolling new drug application (NDA) to the FDA and a marketing authorization application (MAA) to the EMA in October 2016. These applications are for the use of niraparib in the maintenance setting for patients with platinum-sensitive recurrent epithelial 
ovarian, fallopian tube, or primary peritoneal cancer who are in response to platinum-based chemotherapy. Approval would be for use in patients regardless of $B R C A$ and HRD status; this is contrary to the FDA's accelerated approval of olaparib, which has restricted this agent to the $\mathrm{g} B R C A \mathrm{~m}$ population and specifies olaparib as a third-line or later treatment.

Because HR-deficient cancers are expected to be the group most responsive to niraparib therapy-and PARPi overall-the other ongoing niraparib trials are aimed at HRD-positive ovarian cancers. The ENGOT PRIMA study (NCT02655016) is a double-blind randomized (2:1; niraparib: placebo) study of niraparib in the maintenance setting for patients with ovarian cancer who have HRDpositive tumors determined by a centralized HRD test. The primary endpoint is PFS. Patients must have received at least four cycles of a front-line platinum-based regimen with a complete response (CR) or PR and have had a normal or $>90 \%$ decrease in cancer antigen 125 (CA-125). The QUADRA study (NCT02354586), a non-randomized phase II study, will evaluate the safety and efficacy of niraparib in patients with ovarian cancer who have received at least three previous chemotherapy regimens and will test for $\mathrm{g} B R C A \mathrm{~m}$ as well as HRD status. The AVANOVA trial (NCT02354131) is a phase I/II three-arm trial comparing the activity of niraparib alone versus the niraparib-bevacizumab combination versus bevacizumab followed by niraparib in patients with ovarian cancer who are platinum sensitive and HRD positive.

\subsubsection{Rucaparib}

The first PARPi to be tested in humans, rucaparib is an FDA-approved PARP-1, -2 , and -3 inhibitor in an oral formulation (PARP1/2 $K_{\mathrm{i}}$ 1.4/0.17 nM) [22, 48]. Preclinical data suggest that rucaparib has a different enzymatic inhibition profile than the other PARPi; it additionally inhibits tankyrase 1 and 2 as well as PARP-2, -4, -12, -15, and -16 [49]. While these additional targets make rucaparib unique among the currently available PARPi, the clinical relevance of these differences is not clear at this time. In a phase II open-label multicenter trial of rucaparib in patients with $\mathrm{g} B R C A \mathrm{~m}$-associated advanced breast or ovarian cancer, both intermittent and continuous dosing schedules of rucaparib were investigated using pharmacokinetic and pharmacodynamic analysis [21]. The intermittent dosing schedule (5 days of 21), which evaluated intravenous instead of oral administration, was shown to result in an ORR of only $2 \%$, although $41 \%$ of patients (18 of 44 ) achieved stable disease for 12 weeks, and three patients maintained stable disease for $>52$ weeks. The investigators considered the low ORR, in combination with pharmacodynamic data showing recovery of PARP enzyme activity during non-treatment days, to be consistent with a suboptimal dosing schedule, and the study was amended to investigate the efficacy of continuous oral administration. In the oral cohorts, 12 of 13 who were dosed continuously achieved CR/PR or stable disease at 12 weeks (RR $15 \%$ ), with a median duration of response of 179 days (range 84-567). Based on these results, clinical development of rucaparib continued with rucaparib as an oral agent.

A phase I/II study of rucaparib (referred to as 'Study 10') was performed in patients with advanced cancer, including $\mathrm{g} B R C A \mathrm{~m}$-associated ovarian, breast, and pancreatic cancer. In phase I (dose escalation), 56 patients with solid tumors (27 breast, 20 ovarian/peritoneal, 9 other) were enrolled and treated with continuous oral dosing of rucaparib od or bid. In patients with $\mathrm{g} B R C A \mathrm{~m}$-associated ovarian cancer, the disease control rate $(\mathrm{CR}+\mathrm{PR}+\mathrm{SD}$ $\geq 24$ weeks) was $70 \%$ (seven of ten) at doses $\geq 300 \mathrm{mg}$ od. Balancing exposure, tolerability, toxicity, and promising clinical activity, the RP2D was determined to be $600 \mathrm{mg}$ bid [50, 51]. Phase II (NCT01891344) was performed in relapsed heavily pretreated high-grade epithelial ovarian, fallopian tube, or primary peritoneal cancer with $\mathrm{g} B R C A \mathrm{~m}$, and preliminary results have demonstrated an ORR of $65 \%$ [52].

Two important trials are currently ongoing for rucaparib in ovarian cancer: the phase II and phase III ARIEL2 and ARIEL3 (NCT01891344 and NCT01968213, respectively) trials in recurrent ovarian cancer. ARIEL2 is a two-part study; part 1 has been completed and part 2 is currently enrolling. Part 1, a single-arm study of oral rucaparib bid with a primary endpoint of PFS, enrolled patients with recurrent platinum-sensitive high-grade epithelial ovarian, primary peritoneal, or fallopian tube cancer [53, 54]. It aimed to assess the capacity of an integrated biomarker to predict response to rucaparib by prospectively defining three subgroups, using next-generation sequencing (NGS) (performed by the sponsor's partner, Foundation Medicine) to determine the degree of loss of heterozygosity $(\mathrm{LOH})$ as a marker of genomic instability in archival and retreatment biopsies [54-56]. The NGS assay combines somatic BRCA status as well as the percentage of genome-wide $\mathrm{LOH}$ to define these subgroups: $B R C A$ mutant (deleterious germline or somatic), $B R C A \mathrm{WT} / \mathrm{LOH}$ high ( $\mathrm{LOH} \geq 14 \%$ ), or $B R C A \mathrm{WT} / \mathrm{LOH}$ low $(\mathrm{LOH}<14 \%$ ). PFS was longer in rucaparib-treated patients with BRCA-mutant (12.8 months; 95\% CI 9.0-14.7) or BRCA WT/LOH-high platinum-sensitive disease (5.7 months; 95\% CI 5.3-7.6) than in rucaparib-treated patients with $B R C A$ WT/LOHlow carcinomas (5.2 months; 95\% CI 3.6-5.5; $p<0.0001$ for $B R C A$ mutant vs. $B R C A \mathrm{WT} / \mathrm{LOH}$ high; $p=0.011$ for $B R C A$ mutant vs. BRCA WT/LOH low) [54]. Additionally, more patients achieved confirmed RECIST (Response Evaluation Criteria in Solid Tumors) responses in the 
LOH-high subgroup (24 of 82 patients [29\%; 95\% CI 20-40]) than in the LOH-low subgroup (7 of 70 patients [10\%; 95\% CI $4-20 ; p=0.0033]$ ), and the duration of response was longer in the LOH-high subgroup (10.8 months; 95\% CI 5.7-not reached) than in the LOHlow subgroup (5.6 months; 95\% CI 4.6-8.5; $p=0.022$ ) [53]. Interestingly, a recent presentation compared efficacy results between the pre-specified genomic LOH and a 'refined' LOH cut-off point and demonstrated an improvement in the selection of patients most likely to benefit from rucaparib (ORR of $80 \%$ in patients with $B R C A$ mutations for both LOH cut-offs but an improvement in ORR from 35 to $39 \%$ in patients with a BRCA-like LOHhigh signature and from 13 to $14 \%$ in patients without a $B R C A$ mutation or a BRCA-like signature) [57]. Part 2 of ARIEL2 will assess ORR and disease-free survival in rucaparib-treated patients with relapsed disease after three or more lines of chemotherapy. The ongoing ARIEL3 is a trial of rucaparib as switch maintenance therapy after response to platinum-based treatment, with PFS as the primary endpoint. This trial will also attempt to prospectively validate tumor HRD scores using the NGS test noted above (also performed by the trial sponsor's partner, Foundation Medicine) [58].

Rucaparib obtained breakthrough designation from the US FDA in April 2015 as monotherapy for the treatment of advanced ovarian cancer with BRCA mutations, both germline and somatic, in patients who have received at least two lines of prior chemotherapy, based on the results of the two phase II trials noted above [59]. In September 2016, ARIEL4 (NCT02855944) opened to fulfill FDA requirements for a potential accelerated approval of rucaparib. This phase III randomized study of rucaparib versus chemotherapy in patients with relapsed platinum-sensitive gBRCAmonly ovarian cancers who have received at least two lines of chemotherapy is assessing PFS as the primary endpoint and OS as a secondary endpoint. In December 2016, based on the positive results of the ARIEL clinical trials, rucaparib was granted accelerated approval by the FDA [60].

\subsubsection{Talazoparib}

Talazoparib is considered to be the most potent of the PARPi in clinical development, requiring lower concentrations to fully inhibit PARP-1 and -2 (PARP1/2 $K_{\mathrm{i}} 1.2 /$ $0.9 \mathrm{nM})[22,61]$. The preliminary phase I study evaluating this oral agent in advanced solid tumors established the $\mathrm{RP} 2 \mathrm{D}$ as $1 \mathrm{mg} /$ day. This trial included patients with 'deleterious' BRCA1/2 mutations in various tumor types, including ovarian/primary peritoneal, breast, pancreas, and colon. Patients participating in this trial with $\mathrm{g} B R C A \mathrm{~m}$ ovarian tumors had RECIST, CA-125, and clinical benefit responses of 44,70 , and $82 \%$, respectively [48, 61].
Other single-agent talazoparib trials are currently ongoing. Interestingly, this includes a phase 0 trial, POSITION (PilOt Study of InducTion PARP InhibitiON in Ovarian Cancer), which will evaluate the effects of talazoparib on DNA copy number, RNA expression, and protein levels before and after talazoparib treatment (NCT02316834). To further evaluate the pharmacodynamic effects of talazoparib, another ongoing pilot study of talazoparib in solid tumors with deleterious germline and non-germline $B R C A$ mutations is analyzing PARP inhibition and DDR markers in tumor biopsies collected prior to treatment, during cycle 1 , and at disease progression (NCT01989546). A phase I study is also about to be opened that will evaluate talazoparib safety and tolerability and determine the RP2D in patients with liver and kidney dysfunction (NCT02567396). Finally, the single-agent phase III EMBRACA, a two-arm multicenter trial of talazoparib versus physician's choice in patients with advanced and previously treated $\mathrm{g} B R C A \mathrm{~m}$-associated breast cancer, will evaluate PFS as the primary outcome (NCT01945775).

\subsection{Toxicity}

In general, PARPi are very well tolerated. With olaparib, the most commonly reported adverse events (AEs) include nausea (59-78\%), fatigue (41-65\%), vomiting (34-50\%), and anemia (12-32\%) and are predominantly of low-grade intensity [31, 33, 62, 63]. A far more serious event that has been shown to occur is the development of myelodysplastic syndrome (MDS) and transformation to acute myeloid leukemia (AML). The olaparib drug package insert reports that olaparib-related MDS/AML is rare, occurring in $<1 \%$ of patients treated with this agent. However, the majority of secondary MDS/AML cases (17 of 22) in a randomized placebo-controlled trial were fatal, though time on treatment with olaparib in these cases varied from $<6$ months to $>2$ years [64]. Concern about MDS/AML contributed to the delay in the approval of olaparib for a maintenance therapy indication, although a causal relationship with olaparib or any other PARPi therapy has not been conclusively established. All olaparib-associated MDS/AML cases occurred in patients with $\mathrm{g} B R C A \mathrm{~m}$ who were previously treated with DNA-damaging chemotherapy, and it has been postulated that this type of chemotherapy in combination with impaired DNA repair pathways may prime patients to develop MDS/AML.

The dose-limiting toxicity (DLT) profile of veliparib differs slightly from that of olaparib; reported phase I AEs included grade 3 nausea/vomiting at $400 \mathrm{mg}$ bid but also two occurrences of grade 2 seizure at $500 \mathrm{mg}$ bid (in a patient with $\mathrm{g} B R C A \mathrm{~m}$ disease) as well as a grade 2 seizure at $400 \mathrm{mg}$ bid in a non-gBRCAm patient [37]. Reported 
AEs for veliparib in phase II trials are generally low grade and include fatigue (6\%), nausea (4\%), and leukopenia $(2 \%)$, with only one grade 4 occurrence of thrombocytopenia $[38,48,65]$.

The toxicity profile of niraparib differs from that of both olaparib and veliparib; the reported DLTs were fatigue, pneumonitis, and thrombocytopenia in the phase I trial [44]. The published results of the phase III NOVA trial note that the most common $(\geq 10 \%)$ treatment-emergent grade 3/4 AEs in the niraparib arm were thrombocytopenia (33.8\%, without bleeding events), anemia (25.3\%), and neutropenia (19.6\%), resulting in AE-related treatment discontinuation rates of $3.3,1.4$, and $1.9 \%$, respectively [9]. These occurred more commonly in the early cycles of therapy and were resolved with appropriate dose modifications. Of note, the rates of MDS/AML in the niraparib and control arms were similar (1.4 and 1.1\%, respectively).

The largest single-agent trial of rucaparib with comprehensive AE data is ARIEL2 (NCT01891344). The most common treatment-related AEs reported in $\geq 15 \%$ of all patients include nausea, asthenia/fatigue, and treatmentrelated transient elevations in the aspartate transaminase/ alanine transaminase ratio (ALT/AST), which were typically grade 1/2. Reported grade 3/4 treatment-related AEs were anemia $(16 \%)$ and transient ALT/AST elevations $(11 \%)$.

For talazoparib, the original phase I study reported mostly grade 1-2 fatigue, nausea, and alopecia. The other significant toxicity is myelosuppression, especially thrombocytopenia, of grade 3-4. Given its toxicity profile, talazoparib appears to have cytotoxic activity similar to that of chemotherapy [61].

All of the PARPi have the potential to interact with coadministered drugs, and these drug interactions should be avoided whenever possible to limit toxicity. Combinations of olaparib and other myelosuppressive anticancer agents, including DNA-damaging agents, should be avoided, as this may prolong myelosuppressive toxicity. Concomitant use of strong and moderate cytochrome P450 (CYP)-3A inhibitors and inducers should also be avoided, as olaparib is primarily metabolized by the CYP3A enzymes, and coadministration with CYP3A inhibitors or inducers may affect metabolic clearance and alter plasma concentration of the drug [66]. Niraparib also has the potential to interact with CYP metabolism. Niraparib may induce CYP1A2, so concomitant use with drugs that are metabolized by CYP1A2 is not recommended. The drug is also a substrate of the P-glycoprotein (P-gp) transporter, and niraparib clearance can be affected by concurrent administration of P-gp inhibitors or substrates [9]. Furthermore, since thrombocytopenia is common with niraparib and talazoparib, caution should be taken when these agents are coadministered with anticoagulation and antiplatelet drugs
[9, 61]. These are just a few considerations for PARPi administration; further studies will need to be performed for a more complete understanding of the potential drug interactions.

\subsection{In Vitro Companion Diagnostic Assays}

Chromosomal instability, a hallmark of cancer, allows for the accumulation of genetic mutations in the tumor cell genome. These genetic abnormalities can occur in identifiable patterns, including copy number alterations (CNAs), chromosomal translocations, LOH events, microRNAs, or DNA methylation [67]. With the development of highthroughput whole-genome sequencing, it is now possible to detect types of structural disarray or rearrangement and use these signatures to recognize HRD in tumors. These technical developments, and the concept of using these socalled genomic 'scars' to identify tumors that would respond to DNA-damaging agents, has enabled the development and approval of CDx testing for PARPi, as can be seen by the FDA approval of BRACAnalysis ${ }^{\circledR}$ to determine patients eligible to receive treatment with olaparib.

Ironically, it was the results from the negative neoadjuvant trial with iniparib in patients with TNBC that contributed to the concept of predicting response to PARPi treatment through the identification of a genomic-loss signature [68]. The neoadjuvant phase II single-arm trial (prECOG 0105) enrolled patients with stage I-IIIA TNBC or BRCA mutation-associated breast cancer to receive carboplatin, gemcitabine, and iniparib every 21 days for a total of 4-6 cycles. The primary endpoint was pCR, and all patients underwent comprehensive BRCA genotyping; HRD was assessed by a previously validated assay for LOH (HRD-LOH) in pretreatment core breast biopsies. This evaluation was designed to identify HRD independent of the etiology, with a threshold HRD 'score' [69]. Of the 80 patients who received all 6 cycles of therapy, 19 (24\%) had germline BRCA1 or BRCA2 mutations and 66/77 patients had DNA of a quality adequate for extraction and performance of the HRD-LOH assay. Mean HRD-LOH scores were significantly higher in responders than in nonresponders $(p=0.02)$ and remained significantly higher when $B R C A$ germline mutations were excluded. When the data were analyzed using a cut-off of $\geq 10$ as indicative of HRD, responders were more likely to exhibit HRD-LOH scores $\geq 10$ than were non-responders in all patients or the subset of patients who were BRCA WT ( $p=0.0026$ and 0.0024 , respectively). These results demonstrated that a molecularly defined patient subgroup, based on DNA-repair deficiencies rather than specific gene mutations, could be identified for potential PARPi therapy outside of $B R C A$ status. Though iniparib is not relevant to current clinical practice, this assay and later variations have been used as 
the basis for the identification of genomic instability in subsequent PARPi trials and CDx [9, 48].

Though a number of CDx assays have been created, the only one currently approved by the FDA is BRACAnalysis $\mathrm{CDx}^{\circledR}$ (Myriad Genetics), which identifies patients eligible for olaparib using genomic DNA obtained from whole blood specimens collected in EDTA [70]. The BRACAnalysis focuses on $\mathrm{gBRCA} 1 / 2 \mathrm{~m}$ identification through two separate measurements: BRACAnalysis CDx Sanger Sequencing for sequence variants, and BRACAnalysis CDx Large Rearrangement Test $\left(\mathrm{BART}^{\circledR}\right.$ ) for large rearrangements. Exon/intron boundaries of BRCA1/2 (17,337 bases total) for identification of single nucleotide polymorphism (SNP), insertions $\leq 2$ base pairs (bp), and deletions $\leq 5 \mathrm{bp}$ are detected by PCR, followed by Sanger sequencing. This defines the limitations of the test: inability to detect deletions $>5 \mathrm{bp}$, insertions $>2 \mathrm{bp}$, or gene duplication or triplication [71].

Myriad's myChoice $\mathrm{HRD}^{\circledR}$ has been described as an "enhancement" of BRACAnalysis CDx, as it assesses LOH beyond BRCA [48]. The myChoice HRD test uses three independent DNA-based measures of genomic instability: $\mathrm{LOH}$, telomeric allelic imbalance (TAI), and large-scale state transitions (LST). An unweighted sum of these (LOH, TAI, and LST) is used to define a HRD 'score' [72]. The association of HRD (defined as HRD score $\geq 42$ ) or $\mathrm{gBRCA}$ mutation with response to therapy has been validated in three neoadjuvant TNBC trials of platinumcontaining therapy [72], including the neoadjuvant phase II trial of carboplatinum, gemcitabine, and iniparib in TNBC described above. This assay is especially valuable because HRD is detected independent of mechanism (i.e., BRCA related or other) and is highly correlated with multi-etiology defects in BRCA1/2, PTEN, FANCM, and RAD51C [69].

A second CDx has been developed by FoundationOne $^{\mathrm{TM}}$ using formalin-fixed paraffin-embedded (FFPE) archival tissue. This NGS assay uses whole-genome shotgun library construction and hybridization capture with biotinylated DNA oligonucleotides to evaluate 315 cancerrelated genes ( $\geq 4557$ exons) and $\geq 47$ introns of 28 genes, with a gene list that includes BRCA1/2, PALB2, FANCM, BARD1, CHK1, ATM, RAD51C, RAD51B, and BLM [48]. Genetic aberrations tested include CNAs, base substitutions using a Bayesian method, 1-40 bp indels (insertion/ deletions) using the deBruijn approach, rearrangements, and homozygous deletions. Validation testing of this method has been completed in a study using 2221 specimens [73]. Tumors with clinically actionable treatment options were found to have 1579 unique alterations. Specificity exceeded $99 \%$ for all genomic alterations tested: base substitutions $(>99 \%$ when mutant allele frequency $[\mathrm{MAF}] \geq 5 \%$ ), indels ( $>97 \%$ when $\mathrm{MAF} \geq 10 \%$ ),
CNAs (>95\%), and rearrangements (>90\%) [48, 73]. FoundationOne ${ }^{\mathrm{TM}}$ has partnered with Clovis Oncology to develop a CDx in parallel with the clinical development of rucaparib in its ARIEL III study (NCT01968213) as noted above. One important question that arises from the data published to date for both of the CDx tests in development is whether they are sufficiently discriminatory in the identification of patients without germline BRCA mutations who will respond to PARPi. The CDx assays for niraparib and rucaparib are aimed at patients with different stages of disease (though both in platinum-sensitive patients), as niraparib improved PFS in all groups (including the HRDnegative group), whereas clinical differences between LOH-high and -low groups treated with rucaparib do not appear to be clinically relevant [53].

The molecular diagnostic tools used to identify specific tumor characteristics with the intent of predicting an individual's likelihood of response to treatment highlights one of the goals of the modern era of anticancer therapy. The aim is to treat only those patients who will benefit from a specific therapy, limiting the exposure of patients to potential toxicities if there is no predicted clinical benefit. This concept is not new, given that the determination of the presence of the estrogen receptor and its correlation with a positive response to tamoxifen treatment was originally described in the 1970s [74]. The value of the drug-diagnostic codevelopment model has been validated in multiple tumor types (breast, lung, colorectal, melanoma, and others). In light of the pace at which the biotechnology field is developing, it is very likely that incorporating this type of analysis will become routine in therapeutic decision making. Though the current CDx platforms utilize varying genomic approaches, based on either known aberrations (NGS-based or BRACAnalysis) or genomic instability of any etiology (myChoice HRD), it is likely that the next stage of clinical development for PARPi CDx test will aim to expand genetic analysis beyond $B R C A$ status and use more comprehensive tumor analysis to guide treatment decisions.

\section{Resistance to PARP Inhibition}

Molecular mechanisms describing resistance to PARPi are mainly related to the restoration of effective HR. In $B R C A-$ mutated tumors, regaining of $B R C A$ function is considered the primary resistance mechanism. Restoration of $B R C A$ activity can occur through back mutation, reading frame restoration, loss of $B R C A$ promoter methylation, or stabilization of the BRCA1 C-terminal (BRCT) domain by HSP90 function [48, 75, 76]. Loss of 53BP1 is another well-described mechanism of resistance to PARPi. 53BP1 normally prevents the replication protein A (RPA) 
phosphorylation-based DNA damage-repair pathway from restoring single-strand DNA lesions. However, if 53BP1 is lost, RPA can load onto DNA and permit repair, bypassing the need for functional BRCA $[48,77]$.

Preclinical results have also suggested that Schlafen 11 (SLFN11) inactivation may be an additional mechanism of resistance to PARPi [78]. SLFN11 expression has been previously reported as a genomic determinant of response to topoisomerase (Top)-1 inhibitors, Top2 inhibitors, alkylating agents, and DNA synthesis inhibitors, and as a potential predictive biomarker for Top1 inhibitors [79, 80]. SLFN11-deficient cell lines were shown to be resistant to talazoparib therapy, but since SLFN11-deficient cells rely on ataxia-telangiectasia and Rad3-related (ATR) activation for survival when PARP is inhibited, talazoparib resistance could be overcome by combined treatment with the ATR inhibitor VE-821 [78]. While these are only hypothesisgenerating results, they are encouraging and could contribute to our growing understanding of PARPi sensitivity in tumor cells. A currently-enrolling phase I clinical trial of veliparib, VX970 (phosphoATR inhibitor), and low-dose cisplatin (NCT02723864) in patients with solid tumors may further inform this type of combination strategy and may also suggest additional patient populations that can benefit from PARPi.

Additionally, PARP1 expression could be decreased or fully lost due to various regulatory mechanisms, such as promoter hypermethylation, and this reduction in PARP1 results in less PARP trapping [81]. Furthermore, upregulation of $\mathrm{ABC}$ transporters, such as the P-gp efflux pump, is a mechanism of resistance that has been described for multiple targeted agents and can increase PARPi efflux from tumor cells. This increased transporter activity can occur secondary to gene upregulation by promoter fusion [70].

Overall, resistance to PARPi is not unexpected in the context of increased clinical use of these agents, and knowledge and understanding of the relevant molecular mechanisms underlying PARPi resistance will be pertinent and may also lead to novel 'druggable' targets.

\section{Combination Strategies and Trials for PARP Inhibitors}

\subsection{Combining PARPi with Chemotherapy or Radiation}

Preclinically, the combination of PARPi with cytotoxic chemotherapy has been shown to potentiate DNA damage by inhibiting ongoing repair, although combination treatment is associated with an increase in toxicity compared with single-agent chemotherapy. Combinations of PARPi and chemotherapy agents that induce SSBs, such as temozolomide and topoisomerase inhibitors, have been studied [82-84]; combinations with platinum agents have demonstrated the most significant antitumor activity [15]. This 'synergistic' effect may be attributed to the induction of DNA crosslinks by platinum agents, which are usually repaired by HR or BER.

Veliparib has been the most widely studied partner for chemotherapy and radiation. The addition of veliparib to treatment with oral cyclophosphamide, a DNA-alkylating agent that causes DNA crosslinks, did not improve PFS or ORR when compared with reatment with cyclophosphamide alone in BRCA-mutant ovarian cancer [85], but this may be attributable to the low dose of veliparib used in the study $(60 \mathrm{mg}$ od). A second phase I trial of this combination demonstrated that a significantly higher veliparib dose could be combined with cyclophosphamide. A double-blind randomized phase III study evaluating veliparib combined with carboplatin/paclitaxel as maintenance therapy in patients with previously untreated stage III/IV epithelial ovarian cancer is currently recruiting participants (NCT02470585). Veliparib switch maintenance trials are in the planning stages [86]. Several effective veliparib combination trials have been presented or reported since 2015 . Veliparib has been administered in combination with the topoisomerase inhibitor irinotecan, and preliminary results are promising. In an expansion cohort of patients with TNBC in a phase I study of irinotecan and veliparib, seven of eight patients carrying mutations in the BRCA genes had a PR to the combination treatment, and seven of ten patients without known BRCA mutation had disease stabilization as their best response [87]. The dose of veliparib was only $40 \mathrm{mg}$ bid. Data have also been presented from the combination of veliparib and carboplatin/paclitaxel in patients with TNBC [88]. Preliminary phase I results for veliparib in combination with intravenous and intraperitoneal paclitaxel/cisplatin/bevacizumab in the adjuvant setting have achieved a safe RP2D of veliparib $150 \mathrm{mg}$ bid [89]. In the ongoing National Cancer Institute (NCI) trial MPACT (Molecular Profiling based Assignment of Cancer Therapy), veliparib in combination with temozolomide is being evaluated for the treatment of patients carrying genetic defects in the DNA-repair pathway other than $\mathrm{g} B R C A \mathrm{~m}$ [90]. Additionally, and following the growing recognition of the interaction of $B R C A$ genes with many other genes, particularly in the Fanconi anemia (FA) pathway, veliparib was tested alone or in combination with mitomycin C (MMC) in patients with solid tumors and HRD. Patients' tumors were screened for the presence (or lack) of tumor FancD2 nuclear foci formation on their archival tumor material, utilizing a newly developed method (FA triple-stain immunofluorescence [FATSI]). FATSI-negative patients (185/643 patients) were selected 
for enrollment in a two-arm dose-escalation trial of veliparib, or veliparib/ MMC. Of these patients, 61 received veliparib or veliparib/MMC through 14 dose levels. Six antitumor responses occurred, five in the combination arm (three breast, one ovarian, one endometrial [uterine], and one non-small cell lung cancer). Surprisingly, BRCA germline analysis among 51 patients revealed five deleterious mutations, whereas a targeted FA sequencing gene panel (25 genes, selected by a custom library panel using NGS) showed missense/nonsense mutations in 29 of 49 FATSI-negative tumor specimens [91].

However, care must be taken to prevent the onset of AEs when administering such combination treatments. A phase I study of olaparib, cisplatin, and gemcitabine in solid tumors reported grade 4 myelosuppression at the first dose level and needed dose de-escalation to determine the MTD [92]. Veliparib given with topotecan on a 5-day schedule was also associated with significant myelosuppression, limiting the dosage at which veliparib could be safely administered [93], but this combination was tolerated better when topotecan was administered weekly with veliparib (successfully given at doses of at least $200 \mathrm{mg}$ bid) [94].

In comparison with the plethora of combination studies evaluating PARPi in combination with chemotherapy, fewer trials are assessing the potential of PARPi as radiosensitizers. A trial evaluating veliparib with low-dose radiotherapy in patients with peritoneal carcinomatosis reported extended disease stabilization, particularly in ovarian/fallopian tube cancer [95]. Phase I trials of olaparib with radiotherapy for inoperable breast cancer (NCT02227082) and stages II-III laryngeal and human papillomavirus (HPV)-negative oropharyngeal squamous cell carcinomas (NCT02229656) are in progress. The combination of radiotherapy, paclitaxel, and carboplatin with or without veliparib in stage III non-small cell lung cancer is also being evaluated in an ongoing study.

In the case of niraparib, a number of combination trials are ongoing, though none have been reported to date. The phase I ESP1/SARC025 combination trial in Ewing's sarcoma (NCT02044120) is evaluating the safety and MTD of niraparib and temozolomide or irinotecan. The TOPACIO study, part of the Keynote trials (KEYNOTE-162, NCT02657889) is a phase I/II study of niraparib together with the anti-programmed cell death protein 1 (PD-1) immunotherapy agent pembrolizumab in patients with TNBC or ovarian cancer. Once the recommended phase II dose is established, the primary outcome will be the ORR.

No combination trials have yet been reported for rucaparib in reproductive system cancers. An earlier phase II study evaluated intravenous rucaparib and oral temozolomide in 47 patients with chemotherapy-naïve advanced metastatic melanoma [96]. The study met its primary endpoint of improvement in RR, with a noted RR of $17.4 \%$, median time to progression (TTP) of 3.5 months and median OS of 9.9 months, as well as $6 \%$ of patients remaining progression free at 6 months; all of which compared favorably with historical controls. Myelosuppression occurred in 25 patients $(54 \%)$, requiring a $25 \%$ dose reduction for temozolomide.

\subsection{Combining PARPi with Targeted Agents}

A number of completed and ongoing trials have explored or are exploring the clinical impact of combining PARPi with various targeted agents to interrupt cancer-associated signaling pathways. ATM and ATR kinases, as well as other crucial DDR modulators, have been proposed as plausible targets for combination therapy given their role in the DNA-repair pathway. Whole genome sequencing analysis suggests there is a high frequency of gene mutations affecting the activity of these DDR-associated kinases in many tumors [97]. Interestingly, synthetic lethality has been proposed as the underlying mechanism of action for this combinatorial approach, which would potentially be independent of $\mathrm{gBRCAm}$ status [16, 97]. Preliminary xenograft data have also shown more significant anticancer activity with dual PARP and phosphoinositide 3 kinase (PI3K) inhibition [98], and a number of trials combining olaparib with $\mathrm{PI} 3 \mathrm{~K} /$ protein kinase $\mathrm{B}$ (AKT)/mammalian target of rapamycin (mTOR) pathway inhibitors are underway (including the ComPAKT trial, a phase I trial of olaparib in combination with the AKT inhibitor AZD5363 in patients with advanced solid tumors [NCT02338622] and a trial combining olaparib with the PI3K inhibitor buparlisib in patients with TNBC or HGSOC [NCT01623349]).

Combining PARPi with drugs that inhibit tumor angiogenesis is also a promising approach. Olaparib has been tested in combination with cediranib, an oral antiangiogenic agent against vascular endothelial growth factor receptor (VEGFR)-1, -2, and -3 , in both $\mathrm{g} B R C A \mathrm{~m}$ and nonmutated recurrent platinum-sensitive ovarian, primary peritoneal, and fallopian tube cancer [99]. Median PFS, the primary endpoint of the trial, favored the combination in the intent-to-treat population, with PFS of 17.7 months (95\% CI 14.7-not reached) with the combination of olaparib and cediranib versus 9 months (95\% CI 5.7-16.5) in patients treated with olaparib alone (HR 0.42; 95\% CI $0.23-0.76 ; p=0.005)$. In an unplanned exploratory analysis of this trial, the noted improvement in PFS with combination therapy was greater in patients with non-mutated $B R C A$ than in patients with $\mathrm{gBRCAm}$. Not unexpectedly, there was greater incidence of grade 3/4 adverse events, including hypertension, fatigue, and diarrhea in the combination arm, with $77 \%$ of patients requiring a dose reduction compared with $27 \%$ in the olaparib monotherapy 
arm [99]. OS data from this study are awaited. In the meantime, a number of other ongoing trials continue to explore this combinatorial strategy, including in platinumresistant ovarian cancer (NCT02889900) and in both platinum-sensitive and -resistant ovarian cancer that has progressed after receiving PARPi therapy (NCT02681237).

\subsection{Combining PARPi with Immunotherapy}

Immune checkpoint inhibitors block the engagement of specific T-cell signaling pathways by tumor cells. These regulatory pathways typically act to downregulate cytotoxic T-cell activity, and malignant cells can evade the immune response by binding to cell surface receptors. Targeting these mechanisms within the tumor microenvironment may therefore allow cytotoxic $\mathrm{T}$ cells to become activated and initiate antitumor activity [100, 101]. Immune checkpoint blockade is an emerging therapeutic area; however, although the FDA has recently approved several immunotherapies for various solid tumor indications, none have yet been approved for reproductive system cancers.

The checkpoint inhibitors generating the most excitement are those targeting CTLA4 and PD-1/programmed death-ligand 1 (PD-L1). While response rates range from 10 to $30 \%$, marked and durable responses have been observed even after discontinuation of treatment. Further research is required to elucidate which patients will benefit by identifying predictive biomarkers of therapeutic response and to improve efficacy by developing combination immunotherapy strategies that integrate PD-1/PD-L1 antagonists with other biologic and chemotherapeutic agents and radiation therapy. Preclinical data showing the value of combining checkpoint inhibitors with PARPi are accumulating. For example, Higuchi et al. [102] reported synergistic activity in a murine $B R C A l$-deficient ovarian cancer model, including a significant survival benefit, from CTLA-4 blockade therapy administered with PARP inhibition. Another study in this mouse model evaluated the antitumor effects of talazoparib and found they extended beyond the induction of apoptosis; talazoparib administration also resulted in significant increases in the number of peritoneal cluster of differentiation (CD)- $8+\mathrm{T}$ cells and natural killer (NK) cells, as well as in the production of interferon (IFN)- $\gamma$ and tumor necrosis factor (TNF)- $\alpha$, suggesting both intrinsic and extrinsic immunomodulatory effects [103]. This agent, and other PARPi, may have clinical efficacy in combination with immunomodulatory agents in patients who harbor HRD.

Several clinical trials are currently underway evaluating the combination of different PARPi in combination with checkpoint blockade agents (Table 2). Each of these studies is based on preclinical data showing that the combination has a synergistic effect within the tumor microenvironment that potentiates antitumor response. Results from these trials are expected to improve our understanding of how to use these agents and how to select biomarkers for patient selection, leading to better therapeutic options for patients.

\section{Future Directions}

PARPi engender a variety of potent in vitro antitumor effects; they also generate targeted clinical efficacy in $\mathrm{g} B R C A \mathrm{~m}$-associated tumors. Following preclinical observations indicating that these drugs induce synthetic lethality in $\mathrm{g} B R C A \mathrm{~m}$ tumors, there is strong rationale underlying the extensive clinical evaluation of PARPi in tumors driven by the BRCA pathway.

The discovery and development of PARPi is an example of the impact of molecular technology on anticancer therapy, in that molecular studies have facilitated a more precise understanding of cancer and the effects of genomic differences (both somatic and germline) on drug response. The identification of critically important genetic alterations involved in the therapeutic mechanism of action of PARPi, such as in the HR pathway, has enabled the development of predictive biomarkers for these agents. The identification and processing of such biomarkers has led to not only the creation of multiplex CDx but also an expansion in PARPi use in patients with HRD beyond BRCA dependence and likely beyond reproductive system tumors. This is a significant departure from the more conventional selection of chemotherapy based on the histopathological assessment of a tumor. Using biomarker-based diagnosis, especially in early-stage clinical trials, aims to streamline drug development and is changing the paradigm required for FDA approval.

None of the PARPi evaluated in this review have been reported to increase OS to date. Ovarian cancer is one of the very few tumor types in which PFS has been validated as a surrogate marker for OS in the setting of cytotoxic chemotherapy [104], though it is hard to know whether this reference point can be extrapolated to trials of targeted agents [105]. Better PFS outcomes have been heralded as a 'worthy goal' in the oncology community, but controversy still exists as to whether this approach simply lowers the level of evidence demanded to demonstrate clinically significant activity, particularly for targeted agents [105]. The economic burden of administering targeted agents, typically through continuous oral administration, will also need to be considered-though the monthly cost of olaparib would likely compare favorably to the administration of intravenous monoclonal antibodies, such as those against angiogenic growth factors or checkpoint inhibitors. An 
Table 2 Selected ongoing trials evaluating PARP inhibitors in combination with checkpoint inhibitors

\begin{tabular}{|c|c|c|c|c|c|}
\hline $\begin{array}{l}\text { Study number } \\
\text { (clinicaltrials.gov } \\
\text { identifier) }\end{array}$ & Phase & Study agents & Tumor type & Study design & $\begin{array}{l}\text { Primary } \\
\text { outcome } \\
\text { measures }\end{array}$ \\
\hline NCT02571725 & $\mathrm{I} / \mathrm{II}$ & $\begin{array}{l}\text { Olaparib + tremelimumab } \\
\quad(\text { anti-CTLA-4) }\end{array}$ & $\begin{array}{l}B R C A \text {-deficient recurrent } \\
\text { ovarian, fallopian tube, or } \\
\text { primary peritoneal cancer }\end{array}$ & $\begin{array}{l}\text { Single arm; open-label; dose de- } \\
\text { escalation of tremelimumab; } \\
28 \text {-day treatment cycles } \\
\text { Oral olaparib bid on days } \\
1-28+\text { IV tremelimumab once } \\
\text { every } 4 \text { weeks for } 6 \text { doses (then } \\
\text { once every } 12 \text { weeks) }\end{array}$ & $\begin{array}{l}\text { Phase I: } \\
\text { RP2D } \\
\text { Phase II: } \\
\text { ORR }\end{array}$ \\
\hline NCT02849496 & II & $\begin{array}{l}\text { Veliparib }+ \text { atezolizumab } \\
\quad \text { (anti-PD-L1) }\end{array}$ & $\begin{array}{l}\text { Triple-negative breast cancer } \\
\text { (stage III-IV) }\end{array}$ & $\begin{array}{l}\text { Three arms; randomized; open- } \\
\text { label; } 21 \text {-day treatment cycles } \\
\text { Veliparib alone (oral bid on days } \\
\text { 1-21) vs. atezolizumab alone (IV } \\
\text { on day 1) vs. combination }\end{array}$ & PFS \\
\hline NCT02657889 & $\mathrm{I} / \mathrm{II}$ & $\begin{array}{l}\text { Niraparib + pembro- } \\
\text { lizumab (anti-PD-1) }\end{array}$ & $\begin{array}{l}\text { Advanced or metastatic triple- } \\
\text { negative breast cancer or } \\
\text { recurrent ovarian cancer }\end{array}$ & $\begin{array}{l}\text { Single arm; open-label; dose } \\
\text { escalation of niraparib; } 21 \text {-day } \\
\text { treatment cycles } \\
\text { Phase I: ascending doses of oral } \\
\text { niraparib up to } 300 \mathrm{mg} / \text { day on } \\
\text { days } 1-21+\text { IV pembrolizumab } \\
200 \mathrm{mg} \text { on day } 1 \\
\text { Phase II: oral niraparib } \\
\text { (RP2D) + IV pembrolizumab } \\
200 \mathrm{mg} \text { on day } 1\end{array}$ & $\begin{array}{l}\text { Phase I: } \\
\text { RP2D, } \\
\text { DLTs } \\
\text { Phase II: } \\
\text { ORR }\end{array}$ \\
\hline NCT02484404 & $\mathrm{I} / \mathrm{II}$ & $\begin{array}{l}\text { Olaparib and/or } \\
\text { cediranib }+ \text { durvalumab } \\
\text { (MEDI4736; anti-PD-L1) }\end{array}$ & $\begin{array}{l}\text { Advanced solid tumors and } \\
\text { advanced or recurrent } \\
\text { ovarian, triple-negative } \\
\text { breast, lung, prostate, and } \\
\text { colorectal cancers }\end{array}$ & $\begin{array}{l}\text { Multi-arm; non-randomized; open- } \\
\text { label; } 28 \text {-day treatment cycles } \\
\text { Phase I: oral olaparib bid and/or } \\
\text { oral cediranib od + IV } \\
\text { durvalumab once every } 2 \text { weeks } \\
\text { Phase II: oral olaparib } \\
\text { (RP2D) + IV durvalumab once } \\
\text { every } 2 \text { weeks vs. oral cediranib } \\
\text { (RP2D) + IV durvalumab once } \\
\text { every } 2 \text { weeks }\end{array}$ & $\begin{array}{l}\text { Phase I: } \\
\text { RP2D } \\
\text { (advanced } \\
\text { solid } \\
\text { tumors) } \\
\text { Phase II: } \\
\text { ORR } \\
\text { (ovarian } \\
\text { cancer) }\end{array}$ \\
\hline
\end{tabular}

bid twice daily, $D L T$ dose-limiting toxicity, IV intravenous, od once daily, $O R R$ overall response rate, $P D$ - $L 1$ programmed death-ligand 1, $P F S$ progression-free survival, $R P 2 D$ recommended phase II dose

additional factor to consider will be the ancillary costs of managing the respective toxicities that occur with these various therapeutic approaches.

The clinical impact of each of the different PARPi described in this report has not yet been fully realized; as described above, each of these agents has a different biochemical potency and enzymatic profile. Although no direct comparison trials have been undertaken, avenues already exist for such comparisons through the application of pharmacodynamically guided biologically effective dosage regimens in conjunction with validated assays $[65,106]$. There is little doubt that evidence of robust single-agent activity for these agents, especially in the setting of limited therapeutic options for patients with advanced refractory disease, is likely to encourage further effort. The wealth of genomic data obtained from these studies will improve biomarker development and increase knowledge of effective drug-drug combinations, mechanisms of drug resistance, and potential processes of PARP sensitization. The development and regulatory approval of this new class of anticancer agent, and the use of genomic signatures to select patients who will obtain greatest therapeutic benefit, is a significant step forward in providing precision medicine to patients with cancer.

Acknowledgements The authors thank Dr. Laura K. Fogli, Kelly Services, for medical writing support in the preparation of this manuscript.

\section{Compliance with Ethical Standards}

The content of this publication does not necessarily reflect the views or policies of the Department of Health and Human Services, nor does 
mention of trade names, commercial products, or organizations imply endorsement by the US Government.

Funding This project has been funded in whole or in part with federal funds from the National Cancer Institute, National Institutes of Health, under Contract Number HHSN261200800001E.

Conflict of interest GO'Sullivan Coyne, AP Chen, R Meehan, and JH Doroshow have no conflicts of interest.

Open Access This article is distributed under the terms of the Creative Commons Attribution-NonCommercial 4.0 International License (http://creativecommons.org/licenses/by-nc/4.0/), which permits any noncommercial use, distribution, and reproduction in any medium, provided you give appropriate credit to the original author(s) and the source, provide a link to the Creative Commons license, and indicate if changes were made.

\section{References}

1. Jackson SP, Bartek J. The DNA-damage response in human biology and disease. Nature. 2009;461:1071-8.

2. Curtin N. PARP inhibitors for anticancer therapy. Biochem Soc Trans. 2014;42:82-8.

3. Walsh CS. Two decades beyond BRCA1/2: homologous recombination, hereditary cancer risk and a target for ovarian cancer therapy. Gynecol Oncol. 2015;137:343-50.

4. Brown JS, Kaye SB, Yap TA. PARP inhibitors: the race is on. Br J Cancer. 2016;114:713-5.

5. Polyak K, Garber J. Targeting the missing links for cancer therapy. Nat Med. 2011;17:283-4.

6. Kaelin WG Jr. The concept of synthetic lethality in the context of anticancer therapy. Nat Rev Cancer. 2005;5:689-98.

7. Welcsh PL, King MC. BRCA1 and BRCA2 and the genetics of breast and ovarian cancer. Hum Mol Genet. 2001;10:705-13.

8. McCabe N, Turner NC, Lord CJ, Kluzek K, Bialkowska A, Swift $\mathrm{S}$, et al. Deficiency in the repair of DNA damage by homologous recombination and sensitivity to poly(ADP-ribose) polymerase inhibition. Cancer Res. 2006;66:8109-15.

9. Mirza MR, Monk BJ, Herrstedt J, Oza AM, Mahner S, Redondo A, et al. Niraparib maintenance therapy in platinum-sensitive, recurrent ovarian cancer. N Engl J Med. 2016;375(22):2154-64. doi:10.1056/NEJMoa1611310.

10. Sinha G. Downfall of iniparib: a PARP inhibitor that doesn't inhibit PARP after all. J Natl Cancer Inst. 2014;106:djt447.

11. O'Shaughnessy J, Schwartzberg L, Danso MA, Miller KD, Rugo HS, Neubauer M, et al. Phase III study of iniparib plus gemcitabine and carboplatin versus gemcitabine and carboplatin in patients with metastatic triple-negative breast cancer. J Clin Oncol. 2014;32:3840-7.

12. Ba X, Garg NJ. Signaling mechanism of poly(ADP-ribose) polymerase-1 (PARP-1) in inflammatory diseases. Am J Pathol. 2011;178:946-55.

13. Beck C, Robert I, Reina-San-Martin B, Schreiber V, Dantzer F. Poly(ADP-ribose) polymerases in double-strand break repair: focus on PARP1, PARP2 and PARP3. Exp Cell Res. 2014;329:18-25.

14. Loseva O, Jemth AS, Bryant HE, Schuler H, Lehtio L, Karlberg $\mathrm{T}$, et al. PARP-3 is a mono-ADP-ribosylase that activates PARP-1 in the absence of DNA. J Biol Chem. 2010;285:8054-60.

15. Benafif S, Hall M. An update on PARP inhibitors for the treatment of cancer. Onco Targets Ther. 2015;8:519-28.
16. O'Sullivan Coyne G, Chen A, Kummar S. Delivering on the promise: poly ADP ribose polymerase inhibition as targeted anticancer therapy. Curr Opin Oncol. 2015;27:475-81.

17. Rouleau M, Patel A, Hendzel MJ, Kaufmann SH, Poirier GG. PARP inhibition: PARP1 and beyond. Nat Rev Cancer. 2010;10:293-301.

18. Liu X, Shi Y, Maag DX, Palma JP, Patterson MJ, Ellis PA, et al. Iniparib nonselectively modifies cysteine-containing proteins in tumor cells and is not a bona fide PARP inhibitor. Clin Cancer Res. 2012;18:510-23.

19. Helleday T. The underlying mechanism for the PARP and BRCA synthetic lethality: clearing up the misunderstandings. Mol Oncol. 2011;5:387-93.

20. Murai J, Huang SY, Das BB, Renaud A, Zhang Y, Doroshow $\mathrm{JH}$, et al. Trapping of PARP1 and PARP2 by clinical PARP inhibitors. Cancer Res. 2012;72:5588-99.

21. Drew Y, Ledermann J, Hall G, Rea D, Glasspool R, Highley M, et al. Phase 2 multicentre trial investigating intermittent and continuous dosing schedules of the poly(ADP-ribose) polymerase inhibitor rucaparib in germline BRCA mutation carriers with advanced ovarian and breast cancer. $\mathrm{Br} \mathrm{J}$ Cancer. 2016;114:723-30.

22. Shen Y, Aoyagi-Scharber M, Wang B. Trapping poly(ADP-ribose) polymerase. J Pharmacol Exp Ther. 2015;353:446-57.

23. Hartwell LH, Szankasi P, Roberts CJ, Murray AW, Friend SH. Integrating genetic approaches into the discovery of anticancer drugs. Science. 1997;278:1064-8.

24. Nijman SM. Synthetic lethality: general principles, utility and detection using genetic screens in human cells. FEBS Lett. 2011;585:1-6.

25. Farmer $\mathrm{H}$, McCabe $\mathrm{N}$, Lord CJ, Tutt AN, Johnson DA, Richardson TB, et al. Targeting the DNA repair defect in BRCA mutant cells as a therapeutic strategy. Nature. 2005;434:917-21.

26. Fong PC, Boss DS, Yap TA, Tutt A, Wu P, Mergui-Roelvink M, et al. Inhibition of poly(ADP-ribose) polymerase in tumors from BRCA mutation carriers. N Engl J Med. 2009;361:123-34.

27. Fong PC, Yap TA, Boss DS, Carden CP, Mergui-Roelvink M, Gourley C, et al. Poly(ADP)-ribose polymerase inhibition: frequent durable responses in BRCA carrier ovarian cancer correlating with platinum-free interval. J Clin Oncol. 2010;28:2512-9.

28. Audeh MW, Carmichael J, Penson RT, Friedlander M, Powell B, Bell-McGuinn KM, et al. Oral poly(ADP-ribose) polymerase inhibitor olaparib in patients with BRCA1 or BRCA2 mutations and recurrent ovarian cancer: a proof-of-concept trial. Lancet. 2010;376:245-51.

29. Tutt A, Robson M, Garber JE, Domchek SM, Audeh MW, Weitzel JN, et al. Oral poly(ADP-ribose) polymerase inhibitor olaparib in patients with BRCA1 or BRCA2 mutations and advanced breast cancer: a proof-of-concept trial. Lancet. 2010;376:235-44.

30. Gelmon KA, Tischkowitz M, Mackay H, Swenerton K, Robidoux A, Tonkin $\mathrm{K}$, et al. Olaparib in patients with recurrent high-grade serous or poorly differentiated ovarian carcinoma or triple-negative breast cancer: a phase 2, multicentre, open-label, non-randomised study. Lancet Oncol. 2011;12:852-61.

31. Kaye SB, Lubinski J, Matulonis U, Ang JE, Gourley C, Karlan BY, et al. Phase II, open-label, randomized, multicenter study comparing the efficacy and safety of olaparib, a poly (ADPribose) polymerase inhibitor, and pegylated liposomal doxorubicin in patients with BRCA1 or BRCA 2 mutations and recurrent ovarian cancer. J Clin Oncol. 2012;30:372-9.

32. Ledermann J, Harter P, Gourley C, Friedlander M, Vergote I, Rustin $\mathrm{G}$, et al. Olaparib maintenance therapy in platinum-sensitive relapsed ovarian cancer. $\mathrm{N}$ Engl $\mathrm{J}$ Med. 2012;366:1382-92. 
33. Ledermann J, Harter P, Gourley C, Friedlander M, Vergote I, Rustin G, et al. Olaparib maintenance therapy in patients with platinum-sensitive relapsed serous ovarian cancer: a preplanned retrospective analysis of outcomes by BRCA status in a randomised phase 2 trial. Lancet Oncol. 2014;15:852-61.

34. US Food and Drug Administration (FDA). FDA approves Lynparza to treat advanced ovarian cancer [media release]. 19 Dec 2014.

35. AstraZeneca. Lynparza Phase III SOLO-2 trial shows significant progression-free survival benefit [media release]. 26 Oct 2016.

36. AstraZeneca. Lynparza ${ }^{\mathrm{TM}}$ (olaparib) granted Breakthrough Therapy designation by US FDA for treatment of BRCA $1 / 2$ or ATM gene mutated metastatic castration resistant prostate cancer [media release]. 28 Jan 2016.

37. Puhalla S, Beumer JH, Pahuja S, Appleman LJ, Tawbi HA-H, Stoller RG, et al. Final results of a phase 1 study of single-agent veliparib (V) in patients (pts) with either BRCA1/2-mutated cancer $(\mathrm{BRCA}+)$, platinum-refractory ovarian, or basal-like breast cancer (BRCA-wt). In: ASCO Annual Meeting Proceedings; 2014.

38. Coleman RL, Sill MW, Bell-McGuinn K, Aghajanian C, Gray HJ, Tewari KS, et al. A phase II evaluation of the potent, highly selective PARP inhibitor veliparib in the treatment of persistent or recurrent epithelial ovarian, fallopian tube, or primary peritoneal cancer in patients who carry a germline BRCA1 or BRCA2 mutation - an NRG Oncology/Gynecologic Oncology Group study. Gynecol Oncol. 2015;137:386-91.

39. Rugo HS, Olopade OI, DeMichele A, Yau C, van 't Veer LJ, Buxton MB, et al. Adaptive randomization of veliparib-carboplatin treatment in breast cancer. $\mathrm{N}$ Engl $\mathrm{J}$ Med. 2016;375:23-34.

40. Carey LA, Winer EP. I-SPY 2-toward more rapid progress in breast cancer treatment. N Engl J Med. 2016;375:83-4.

41. Cortazar P, Zhang L, Untch M, Mehta K, Costantino JP, Wolmark N, et al. Pathological complete response and long-term clinical benefit in breast cancer: the $\mathrm{CTNeoBC}$ pooled analysis. Lancet. 2014;384:164-72.

42. Meehan RS, Chen AP, Doroshow JH. Role of adaptive randomization in developing novel therapies for patients with breast cancer. Transl Cancer Res. 2016;5:S1119-22.

43. Konecny GE, Kristeleit RS. PARP inhibitors for BRCA1/2mutated and sporadic ovarian cancer: current practice and future directions. Br J Cancer. 2016;115(10):1157-73. doi:10.1038/bjc. 2016.311.

44. Sandhu SK, Schelman WR, Wilding G, Moreno V, Baird RD, Miranda S, et al. The poly(ADP-ribose) polymerase inhibitor niraparib (MK4827) in BRCA mutation carriers and patients with sporadic cancer: a phase 1 dose-escalation trial. Lancet Oncol. 2013;14:882-92.

45. Burgess M, Puhalla S. BRCA 1/2-mutation related and sporadic breast and ovarian cancers: more alike than different. Front Oncol. 2014;4:19.

46. Cancer Genome Atlas Research N. Integrated genomic analyses of ovarian carcinoma. Nature. 2011;474:609-15.

47. Etemadmoghadam D, Weir BA, Au-Yeung G, Alsop K, Mitchell G, George J, et al. Synthetic lethality between CCNE1 amplification and loss of BRCA1. Proc Natl Acad Sci. 2013;110:19489-94.

48. Jenner ZB, Sood AK, Coleman RL. Evaluation of rucaparib and companion diagnostics in the PARP inhibitor landscape for recurrent ovarian cancer therapy. Future Oncol. 2016;12:1439-56.

49. Wahlberg E, Karlberg T, Kouznetsova E, Markova N, Macchiarulo A, Thorsell AG, et al. Family-wide chemical profiling and structural analysis of PARP and tankyrase inhibitors. Nat Biotechnol. 2012;30:283-8.
50. Kristeleit R, Shapira-Frommer R, Burris H, Patel M, Lorusso P, Oza A, et al. Phase 1/2 study of oral rucaparib: updated phase 1 and preliminary phase 2 results. Ann Oncol. 2014;25:iv307-8.

51. Kristeleit RS, Shapiro G, LoRusso P, Infante JR, Flynn M, Patel MR, et al. A phase I dose-escalation and PK study of continuous oral rucaparib in patients with advanced solid tumors. In: ASCO Annual Meeting Proceedings; 2013.

52. Shapira-Frommer R, Oza AM, Domchek SM, Balmana J, Patel MR, Chen L-M, et al. A phase II open-label, multicenter study of single-agent rucaparib in the treatment of patients with relapsed ovarian cancer and a deleterious BRCA mutation. In: ASCO Annual Meeting Proceedings; 2015.

53. González Martin A. Progress in PARP inhibitors beyond BRCA mutant recurrent ovarian cancer? Lancet Oncol. 2016. doi:10. 1016/S1470-2045(16)30621-0 (Epub 28 Nov 2016).

54. Swisher EM, Lin KK, Oza AM, Scott CL, Giordano H, Sun J, et al. Rucaparib in relapsed, platinum-sensitive high-grade ovarian carcinoma (ARIEL2 part 1): an international, multicentre, open-label, phase 2 trial. Lancet Oncol. 2016. doi:10. 1016/S1470-2045(16)30559-9 (Epub 28 Nov 2016).

55. Lin K, Sun J, Maloney L, Goble S, Oza A, Coleman R, et al. 2701 quantification of genomic loss of heterozygosity enables prospective selection of ovarian cancer patients who may derive benefit from the PARP inhibitor rucaparib. Eur J Cancer. 2015;51:S531-2.

56. Sun JX, Frampton G, Wang K, Ross JS, Miller VA, Stephens PJ, et al. A computational method for somatic versus germline variant status determination from targeted next-generation sequencing of clinical cancer specimens without a matched normal control. Cancer Res. 2014;74:1893.

57. Coleman RL, Swisher EM, Oza AM, Scott CL, Giordano H, Lin $\mathrm{KK}$, et al. Refinement of prespecified cutoff for genomic loss of heterozygosity (LOH) in ARIEL2 part 1: a phase II study of rucaparib in patients (pts) with high grade ovarian carcinoma (HGOC). In: ASCO Annual Meeting Proceedings; 2016.

58. Swisher EM, McNeish IA, Coleman RL, Brenton J, Kaufmann $\mathrm{SH}$, Allen AR, et al. ARIEL 2/3: an integrated clinical trial program to assess activity of rucaparib in ovarian cancer and to identify tumor molecular characteristics predictive of response. In: ASCO Annual Meeting Proceedings; 2014.

59. Clovis Oncology, Inc. Clovis oncology receives breakthrough therapy designation for rucaparib for monotherapy treatment of advanced ovarian cancer in patients with BRCA-mutated tumors (inclusive of both germline and somatic BRCA mutations) [media release]. 6 Apr 2015.

60. US Food and Drug Administration (FDA). FDA grants accelerated approval to new treatment for advanced ovarian cancer [media release]. 19 Dec 2016.

61. De Bono JS, Mina LA, Gonzalez M, Curtin NJ, Wang E, Henshaw JW, et al. First-in-human trial of novel oral PARP inhibitor BMN 673 in patients with solid tumors. In: ASCO Annual Meeting Proceedings; 2013.

62. Bixel K, Hays JL. Olaparib in the management of ovarian cancer. Pharmgenomics Pers Med. 2015;8:127-35.

63. Kaufman B, Shapira-Frommer R, Schmutzler RK, Audeh MW, Friedlander $\mathrm{M}$, Balmana $\mathrm{J}$, et al. Olaparib monotherapy in patients with advanced cancer and a germline BRCA1/2 mutation. J Clin Oncol. 2015;33:244-50.

64. Ricks TK, Chiu HJ, Ison G, Kim G, McKee AE, Kluetz P, et al. Successes and challenges of PARP inhibitors in cancer therapy. Front Oncol. 2015;5:222.

65. Kummar S, Kinders R, Gutierrez ME, Rubinstein L, Parchment RE, Phillips LR, et al. Phase 0 clinical trial of the poly (ADPribose) polymerase inhibitor ABT-888 in patients with advanced malignancies. J Clin Oncol. 2009;27:2705-11. 
66. Lynparza ${ }^{\mathrm{TM}}$ (olaparib) [package insert]. Wilmington: AstraZeneca Pharmaceuticals LP; 2016.

67. Lord CJ, Ashworth A. BRCAness revisited. Nat Rev Cancer. 2016;16:110-20.

68. Telli ML, Jensen KC, Vinayak S, Kurian AW, Lipson JA, Flaherty PJ, et al. Phase II study of gemcitabine, carboplatin, and iniparib as neoadjuvant therapy for triple-negative and BRCA1/ 2 mutation-associated breast cancer with assessment of a tumorbased measure of genomic instability: PrECOG 0105. J Clin Oncol. 2015;33:1895-901.

69. Abkevich V, Timms KM, Hennessy BT, Potter J, Carey MS, Meyer LA, et al. Patterns of genomic loss of heterozygosity predict homologous recombination repair defects in epithelial ovarian cancer. Br J Cancer. 2012;107:1776-82.

70. Bouwman P, Jonkers J. Molecular pathways: how can BRCAmutated tumors become resistant to PARP inhibitors? Clin Cancer Res. 2014;20:540-7.

71. Gunderson CC, Moore KN. BRACAnalysis CDx as a companion diagnostic tool for Lynparza. Expert Rev Mol Diagn. 2015;15:1111-6.

72. Telli ML, Timms KM, Reid J, Hennessy B, Mills GB, Jensen $\mathrm{KC}$, et al. Homologous recombination deficiency (HRD) score predicts response to platinum-containing neoadjuvant chemotherapy in patients with triple-negative breast cancer. Clin Cancer Res. 2016;22:3764-73.

73. Frampton GM, Fichtenholtz A, Otto GA, Wang K, Downing SR, $\mathrm{He} \mathrm{J}$, et al. Development and validation of a clinical cancer genomic profiling test based on massively parallel DNA sequencing. Nat Biotechnol. 2013;31:1023-31.

74. Lerner HJ, Band PR, Israel L, Leung BS. Phase II study of tamoxifen: report of 74 patients with stage IV breast cancer. Cancer Treat Rep. 1976;60:1431-5.

75. Dhillon KK, Swisher EM, Taniguchi T. Secondary mutations of BRCA1/2 and drug resistance. Cancer Sci. 2011;102:663-9.

76. Meehan RS, Chen AP. New treatment option for ovarian cancer: PARP inhibitors. Gynecol Oncol Res Pract. 2016;3:3.

77. Bunting SF, Callen E, Wong N, Chen HT, Polato F, Gunn A, et al. 53BP1 inhibits homologous recombination in Brca1-deficient cells by blocking resection of DNA breaks. Cell. 2010;141:243-54.

78. Murai J, Feng Y, Yu GK, Ru Y, Tang SW, Shen Y, et al. Resistance to PARP inhibitors by SLFN11 inactivation can be overcome by ATR inhibition. Oncotarget. 2016. doi:10.18632/ oncotarget.12266(Epub 27 Sep 2016).

79. Nogales V, Reinhold WC, Varma S, Martinez-Cardus A, Moutinho C, Moran S, et al. Epigenetic inactivation of the putative DNA/RNA helicase SLFN11 in human cancer confers resistance to platinum drugs. Oncotarget. 2016;7:3084-97.

80. Barretina J, Caponigro G, Stransky N, Venkatesan K, Margolin AA, Kim S, et al. The cancer cell line encyclopedia enables predictive modelling of anticancer drug sensitivity. Nature. 2012;483:603-7.

81. Liu X, Han EK, Anderson M, Shi Y, Semizarov D, Wang G, et al. Acquired resistance to combination treatment with temozolomide and ABT- 888 is mediated by both base excision repair and homologous recombination DNA repair pathways. Mol Cancer Res. 2009;7:1686-92.

82. Smith MA, Reynolds CP, Kang MH, Kolb EA, Gorlick R, Carol $\mathrm{H}$, et al. Synergistic activity of PARP inhibition by talazoparib (BMN 673) with temozolomide in pediatric cancer models in the pediatric preclinical testing program. Clin Cancer Res. 2015;21:819-32.

83. Zaremba T, Curtin NJ. PARP inhibitor development for systemic cancer targeting. Anticancer Agents Med Chem. 2007;7:515-23.
84. Xu Y, Her C. Inhibition of topoisomerase (DNA) I (TOP1): DNA damage repair and anticancer therapy. Biomolecules. 2015;5:1652-70.

85. Kummar S, Oza AM, Fleming GF, Sullivan DM, Gandara DR, Naughton MJ, et al. Randomized trial of oral cyclophosphamide and veliparib in high-grade serous ovarian, primary peritoneal, or fallopian tube cancers, or BRCA-mutant ovarian cancer. Clin Cancer Res. 2015;21:1574-82.

86. Khalique S, Hook JM, Ledermann JA. Maintenance therapy in ovarian cancer. Curr Opin Oncol. 2014;26:521-8.

87. LoRusso PM, Li J, Burger A, Heilbrun LK, Sausville EA, Boerner SA, et al. Phase I safety, pharmacokinetic, and pharmacodynamic study of the poly(ADP-ribose) polymerase (PARP) inhibitor veliparib (ABT-888) in combination with irinotecan in patients with advanced solid tumors. Clin Cancer Res. 2016;22:3227-37.

88. Pahuja S, Beumer JH, Appleman LJ, Tawbi HA-H, Stoller RG, Lee JJ, et al. A phase I study of veliparib (ABT-888) in combination with weekly carboplatin and paclitaxel in advanced solid malignancies and enriched for triple-negative breast cancer (TNBC). In: ASCO Annual Meeting Proceedings; 2015.

89. Bell-McGuinn KM, Brady WE, Schilder RJ, Fracasso PM, Moore KN, Walker JL, et al. A phase I study of continuous veliparib in combination with IV carboplatin/paclitaxel or IV/IP paclitaxel/cisplatin and bevacizumab in newly diagnosed patients with previously untreated epithelial ovarian, fallopian tube, or primary peritoneal cancer: an NRG Oncology/Gynecologic Oncology Group study. In: ASCO Annual Meeting Proceedings; 2015.

90. Do K, O'Sullivan Coyne G, Chen AP. An overview of the NCI precision medicine trials-NCI MATCH and MPACT. Chin Clin Oncol. 2015;4:31.

91. Villalona-Calero MA, Duan W, Zhao W, Shilo K, Schaaf LJ, Thurmond $\mathrm{J}$, et al. Veliparib alone or in combination with mitomycin $\mathrm{C}$ in patients with solid tumors with functional deficiency in homologous recombination repair. J Natl Cancer Inst. 2016;108:djv437.

92. Rajan A, Carter CA, Kelly RJ, Gutierrez M, Kummar S, Szabo E, et al. A phase I combination study of olaparib with cisplatin and gemcitabine in adults with solid tumors. Clin Cancer Res. 2012;18:2344-51.

93. Kummar S, Chen A, Ji J, Zhang Y, Reid JM, Ames M, et al. Phase I study of PARP inhibitor ABT-888 in combination with topotecan in adults with refractory solid tumors and lymphomas. Cancer Res. 2011;71:5626-34.

94. Wahner Hendrickson AE, Menefee ME, Hartmann LC, Long HJ, Northfelt DW, Reid JM, et al. A phase I trial of veliparib, an inhibitor of poly (ADP-ribose) polymerase (PARP), and topotecan (TPT) in patients with solid tumors. J Clin Oncol 2015;33(Suppl):abstr TPS2618.

95. Reiss KA, Herman JM, Zahurak M, Brade A, Dawson LA, Scardina A, et al. A phase I study of veliparib (ABT-888) in combination with low-dose fractionated whole abdominal radiation therapy in patients with advanced solid malignancies and peritoneal carcinomatosis. Clin Cancer Res. 2015;21:68-76.

96. Plummer R, Lorigan P, Steven N, Scott L, Middleton MR, Wilson RH, et al. A phase II study of the potent PARP inhibitor, rucaparib (PF-01367338, AG014699), with temozolomide in patients with metastatic melanoma demonstrating evidence of chemopotentiation. Cancer Chemother Pharmacol. 2013;71:1191-9.

97. Weber AM, Ryan AJ. ATM and ATR as therapeutic targets in cancer. Pharmacol Ther. 2015;149:124-38.

98. Juvekar A, Burga LN, Hu H, Lunsford EP, Ibrahim YH, Balmana J, et al. Combining a PI3K inhibitor with a PARP inhibitor 
provides an effective therapy for BRCA1-related breast cancer. Cancer Discov. 2012;2:1048-63.

99. Liu JF, Barry WT, Birrer M, Lee JM, Buckanovich RJ, Fleming GF, et al. Combination cediranib and olaparib versus olaparib alone for women with recurrent platinum-sensitive ovarian cancer: a randomised phase 2 study. Lancet Oncol. 2014; 15:1207-14

100. Pardoll DM. The blockade of immune checkpoints in cancer immunotherapy. Nat Rev Cancer. 2012;12:252-64.

101. Korman AJ, Peggs KS, Allison JP. Checkpoint blockade in cancer immunotherapy. Adv Immunol. 2006;90:297-339.

102. Higuchi T, Flies DB, Marjon NA, Mantia-Smaldone G, Ronner L, Gimotty PA, et al. CTLA-4 blockade synergizes therapeutically with PARP inhibition in BRCA1-deficient ovarian cancer. Cancer Immunol Res. 2015;3:1257-68.
103. Huang J, Wang L, Cong Z, Amoozgar Z, Kiner E, Xing D, et al. The PARP1 inhibitor BMN 673 exhibits immunoregulatory effects in a Brca1(-/-) murine model of ovarian cancer. Biochem Biophys Res Commun. 2015;463:551-6.

104. Bast RC, Thigpen JT, Arbuck SG, Basen-Engquist K, Burke LB, Freedman R, et al. Clinical trial endpoints in ovarian cancer: report of an FDA/ASCO/AACR Public Workshop. Gynecol Oncol. 2007;107:173-6.

105. Booth CM, Eisenhauer EA. Progression-free survival: meaningful or simply measurable? J Clin Oncol. 2012;30:1030-3.

106. Parchment RE, Doroshow JH. Pharmacodynamic endpoints as clinical trial objectives to answer important questions in oncology drug development. Semin Oncol. 2016;43:514-25. 